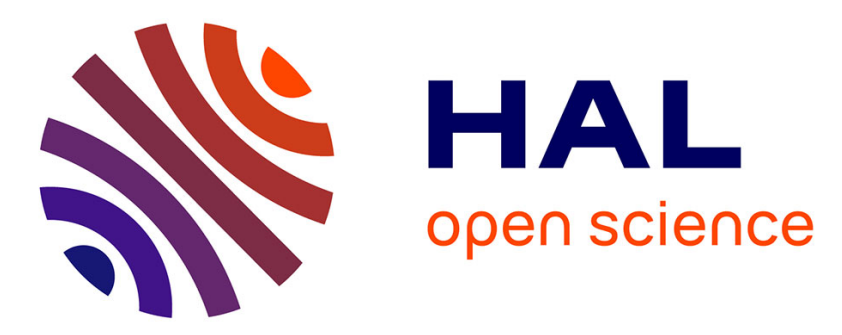

\title{
Détection d'hélicoptères par analyse du bruit acoustique rayonné par leur rotor principal
}

\author{
M. Pecot, G. Vezzosi, F. Ehrmann
}

\section{To cite this version:}

M. Pecot, G. Vezzosi, F. Ehrmann. Détection d'hélicoptères par analyse du bruit acoustique rayonné par leur rotor principal. Journal de Physique IV Proceedings, 1994, 04 (C5), pp.C5-1339-C5-1342. 10.1051/jp4:19945298 . jpa-00253011

\section{HAL Id: jpa-00253011 https://hal.science/jpa-00253011}

Submitted on 1 Jan 1994

HAL is a multi-disciplinary open access archive for the deposit and dissemination of scientific research documents, whether they are published or not. The documents may come from teaching and research institutions in France or abroad, or from public or private research centers.
L'archive ouverte pluridisciplinaire HAL, est destinée au dépôt et à la diffusion de documents scientifiques de niveau recherche, publiés ou non, émanant des établissements d'enseignement et de recherche français ou étrangers, des laboratoires publics ou privés. 


\title{
Détection d'hélicoptères par analyse du bruit acoustique rayonné par leur rotor principal
}

\author{
M. PECOT, G. VEZZOSI ${ }^{*}$ et F. EHIRMANN*
}

Thomson-CSF/LER, Avenue de Belle Fontaine, 35510 Cesson-Sévigné, France

* LTSI, Université de Rennes I, Campus de Beaulieu, 35042 Rennes cedex, France

\begin{abstract}
This paper describes a technique for the detection of helicopters based on the farfield acoustic signal produced by their main rotor. This signal may be well modelled as that produced by a point-like rotating source with periodic excitation. The corresponding harmonics may be computed analytically, which allows to define a receiver using both the modulus and the phase of the measured harmonics. Such a receiver may then be considered as a variant of the matched filter, which improves detection capabilities and allows for some rough classification of the detected signal.
\end{abstract}

\section{1 - Introduction}

Cette communication décrit une technique de détection et classification d'hélicoptères par des moyens acoustiques passifs, à partir d'une analyse fine du bruit rayonné par leur rotor principal. Plusieurs raisons conduisent à privilégier ce signal : d'une part il s'agit d'un signal basse-fréquence $(10-300 \mathrm{~Hz})$, donc peu atténué par la propagation. D'autre part il est approximativement périodique et de nature impulsive en raison de l'effet Doppler produit par la rotation, ce qui permet de le discriminer vis à vis de bruiteurs parasites. Le développement d'un récepteur performant passe néanmoins par une meilleure connaissance du signal à détecter afin d'exploiter autre chose que sa seule pseudo-périodicité. Cependant, la forme du signal varie beaucoup en fonction des conditions de vol, et les prévisions théoriques qui pourraient être obtenues en intégrant les équations de l'acoustique le long de la pale sont trop complexes pour permettre de spécifier un détecteur. En pratique, elles servent essentiellement à calculer des profils de pale permettant de réduire le bruit rayonné.

Dans ce contexte, la description du signal rayonné à l'aide de l'ancien modèle de Gutin permet, malgré sa simplicité, de spécifier un détecteur possédant des performances tout à fait honorables, en tous cas bien supérieures à celles d'une détection ne prenant en compte que la périodicité du signal (harmogramme d'Hinich). Le récepteur obtenu autorise par ailleurs une pré-classification des hélicoptères en fonction de leur nombre de pales et de leur nombre de Mach.

Après avoir rappelé la démarche qui conduit aux expressions analytiques des harmoniques de la pression acoustique dans le cadre du modèle de Gutin, le récepteur est défini, puis ses performances sont comparées au récepteur incohérent basé sur l'harmogramme d'Hinich. 


\section{2 - Le modèle de Gutin}

L'objet de ce modèle mathématique est de conduire à une description suffisamment fidèle de la partie basse fréquence du signal de pression acoustique généré à grande distance par les pales d'un hélicoptère. Chaque pale est assimilée à une source ponctuelle tournante placée à son extrémité et soumise à des excitations périodiques, décrites par leurs coefficients de Fourier. Ces lois d'excitation rendent compte des diverses forces s'exerçant sur la pale en rotation.

Pour un hélicoptère immobile, le signal de pression produit par un pale est solution de l'équation :

$$
\Delta p-\frac{1}{c^{2}} \cdot \frac{\partial^{2} p}{\partial t^{2}}=-\frac{\partial}{\partial t}(Q(\Omega t) \cdot \delta(\vec{r}-\zeta(t))+\operatorname{div}(F(\Omega t) \delta(\vec{r}-\zeta(t))
$$

où $\mathrm{c}$ est la célérité du son dans l'air, le vecteur $\zeta(t)=(R \cos \Omega t, R \sin \Omega t, 0)$ est la trajectoire de l'extrémité de la pale de rayon $R$ et vitesse de rotation $\Omega$. Au second membre, la dérivée temporelle représente le bruit d'épaisseur de la pale, résultant du débit masse $Q(\Omega t)$ engendré par la pale. La divergence représente le bruit de charge, produit par la force $F(\Omega t)$ qu'elle exerce sur l'air ambiant. Un troisième terme existe en pratique (bruit de cisaillement), faisant intervenir le tenseur de Lighthill [1], néanmoins ce terme n'est significatif qu'aux nombres de Mach $(M=R \Omega / c)$ très proches de 1 et peut être négligé en première approximation.

Dans ce qui suit, $r, \psi$ et $\theta$ représentent les coordonnées sphériques du récepteur dans le repère lié à l'hélicoptère, et $\mathrm{M}_{\psi}=\mathrm{M} \cdot \cos \psi$. L'équation précédente se résout en dérivant par rapport au temps et aux variables spatiales le potentiel $\Phi(r, t)$ solution d'une équation du type :

$$
\Delta \Phi-\frac{1}{c^{2}} \cdot \frac{\partial^{2} \Phi}{\partial t^{2}}=-q(\Omega t) \cdot \delta(\vec{r}-\zeta(t))
$$

A très grande distance $(r \gg R$ ) et pour une vitesse subsonique, on montre [2] que $\Phi(r, t)$ admet comme fonction de $t$ la période $T=2 \pi / \Omega$, et s'exprime sous la forme :

$$
\Phi(\vec{r}, t)=\frac{1-\partial \tau / \partial t}{4 \pi r} \cdot q(\Omega(t-\tau))
$$

où $\tau(\mathrm{t})$ est le temps de propagation du signal émis par la source, ce qui permet d'obtenir son développement en série de Fourier :

$$
\Phi(\vec{r}, t)=\sum_{n} c_{n} e^{j n \Omega(t-\pi(c)+j n(\pi / 2-\theta)} \text { avec } c_{n}=\frac{1}{8 \pi^{2} r} \int_{-\pi}^{\pi} q(u+\theta-\pi / 2) \cdot e^{-j n(u-M / s \sin u)} d u
$$

Dans le cas d'une source tournante en translation à la vitesse radiale $\mathrm{v}_{\mathrm{r}}$, la relation précédente subsiste à condition de remplacer $\Omega$ par $\Omega /\left(1-v_{r} / c\right)$ (y compris dans le nombre de Mach) et de multiplier les coefficients de Fourier $c_{n}$ par le facteur de convection dû à la translation $1 /\left(1-v_{f} / c\right)$.

Le signal de pression acoustique est la somme des signaux d'épaisseur et de charge. Le premier s'obtient en remplaçant $\mathrm{q}(\mathrm{x})$ par $\mathrm{Q}(\mathrm{x})$ dans la solution précédente et en dérivant ensuite la fonction $\Phi$ par rapport au temps. Le second terme s'obtient en remplaçant successivement $q(x)$ par les composantes cartésiennes de la force $F$, en prenant les dérivées des 3 fonctions $\Phi$ obtenues en $x, y$ et $z$ et en sommant les 3 contributions. Le calcul des 
dérivées montre que le bruit de charge ne dépend en fait que de la composante radiale de la force. Il apparait alors que les bruits d'épaisseur et de charge ont analytiquement les mêmes expressions, si bien que le $\mathrm{n}^{\text {ième }}$ harmonique de pression s'écrit sous la forme :

$$
c_{n}=\sum_{m} A_{m} \cdot \alpha(m, n, M)
$$

où les $\mathrm{A}_{\mathrm{m}}$ sont des coefficients complexes (inconnus) directement reliés aux coefficients de Fourier des lois d'excitation (harmoniques de charge), et $\alpha(\mathrm{m}, \mathrm{n}, \mathrm{M})$ des expressions intégrales faisant intervenir le nombre de Mach de la pale.

Le cas d'un rotor à $B$ pales se traite immédiatement : on est conduit à faire la somme de $B$ signaux identiques de période $\mathrm{T}$, décalés de $\mathrm{T} / \mathrm{B}$ l'un par rapport à l'autre : les harmoniques d'indice non nultiple de B s'annulent, tandis que les autres sont multipliés par B.

\section{3 - Définition du récepteur et performances}

Les relations précédentes montrent que les harmoniques du signal de pression $\left\{c_{n}\right\}$ s'expriment comme des combinaisons linéaires des harmoniques de charge $\left\{A_{m}\right\}$, les coefficients de la décomposition étant indexés par le nombre de Mach et le nombre de pales. Supposons provisoirement connus le fondamental $f_{0}$, ainsi que $B$ et $M_{\psi}$. La détection de présence d'un hélicoptère se ramène alors [3],[4] au test des 2 hypothèses :

$$
\begin{aligned}
& H_{0}: f_{n}=b_{n} \\
& H_{1}: f_{n}=c_{n} \cdot e^{j n \varphi}+b_{n}
\end{aligned}
$$

où $\left\{f_{n}\right\}$ est le vecteur des coefficients de Fourier obtenu par transformée de Fourier du signal reçu, $\left\{b_{n}\right\}$ un bruit blanc gaussien circulaire. Le terme de déphasage rend compte du retard entre le signal mesuré et son modèle.

Les essais sur signaux réels ont montré que $90 \%$ de la puissance du signal est produite par les deux premiers harmoniques de charge $A_{0}$ et $A_{1}$. Dans ces conditions, le vecteur des coefficients de Fourier du signal de pression peut être considéré comme élément d'un espace vectoriel à 2 dimensions, et on est ramené à la détection d'un signal connu à un facteur et un déphasage près. En utilisant une technique de maximum de vraisemblance, le test se met alors sous la forme :

$$
R=\sup _{\varphi}\left\{\left|\underline{u^{t}} \cdot \operatorname{Ref}(\varphi)\right|^{2}+\left|\underline{v^{t}} \cdot \operatorname{Im} \underline{f}(\varphi)\right|^{2}\right\}
$$

où les vecteurs $\underline{\underline{u}}$ et $\underline{\mathrm{v}}$ sont les deux vecteurs de base du sous-espace vectoriel décrivant les harmoniques de pression. Ces deux vecteurs s'expriment simplement en fonction des intégrales $\alpha(m, n, M)$. On décide $H_{1}$ si $R$ est supérieur à un seuil défini en fonction de la probabilité de fausse alarme souhaitée, $\mathrm{H}_{0}$ dans le cas contraire.

La maximisation en $\varphi$ se fait par un calcul de transformée de Fourier. Elle doit être complétée par une maximisation sur $\mathrm{f}_{0}, \mathrm{~B}$ et $\mathrm{M}_{\psi}$, puisque ces paramètres sont inconnus. Si on admet que $B$ varie entre 2 et 5 et $M_{\psi}$ entre 0.4 et 0.9 par pas de 0.1 , on doit alors traiter 24 couples $\left(\mathrm{B}, \mathrm{M}_{\psi}\right)$. Cependant, certains couples conduisent à des vecteurs $\underline{\mathrm{u}}$ et $\underline{\mathrm{v}}$ pratiquement colinéaires. Une procédure de classification [3] destinée à réduire le nombre de sous-espaces permet de ramener le nombre de couples traités à 3 .

Le récepteur ainsi défini permet donc la détection de présence d'un hélicoptère et une 
pre-discrimination de son couple $\left(B, M_{\psi}\right)$ selon 3 classes. Ses performances ont été analysées sur un ensemble de signaux réels issus de la campagne d'expérimentation AMI2 de la SEFT. Cette analyse a porté sur la comparaison des courbes COR de ce récepteur et de celles de l'harmogramme d'Hinich pondéré ou non. Rappelons que ce dernier récepteur est défini par:

$$
R_{1}=\sum_{n=1}^{N} \sigma_{n}^{2}\left|f_{n}\right|^{2}
$$

avec $\sigma_{\mathrm{n}}{ }^{2}=1 \forall \mathrm{n}$ sans pondération. Une loi de décroissance de $4 \mathrm{~dB}$ par harmonique a été retenue pour le récepteur pondéré $\left(\sigma_{n+1}^{2}=\sigma_{n}^{2} / 2.5\right)$. Ces courbes COR, mesurées pour un rapport $\mathrm{S} / \mathrm{B}$ temporel de $-20 \mathrm{~dB}$, sont représentées figure 1 et montrent clairement la supériorité du récepteur développé. Il offre de plus une meilleure réjection des brouilleurs périodiques dont les harmoniques ne respectent pas la loi de phase du modèle.

D'autre part, cette analyse a permis de mettre en évidence un gain de l'ordre de $5 \mathrm{~dB}$ en terme de rapport S/B pour la détection, par rapport à l'harmogramme classique.
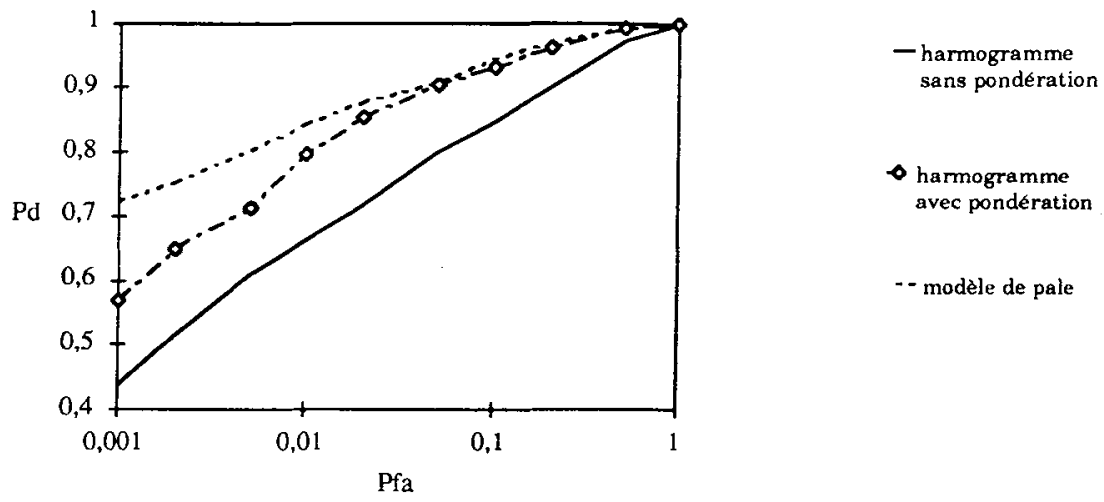

Figure 1 : Courbes COR des 3 récepteurs

\section{4-Conclusion}

L'analyse précédente a mis en évidence le bénéfice que l'on peut tirer de la connaissance de la loi de phase des harmoniques pour la détection d'un signal périodique, le récepteur devenant alors une variante du filtre adapté. Appliquée au cas de la détection acoustique d'hélicoptères, cette approche permet d'améliorer la portée de détection et conduit à une meilleure réjection des brouilleurs périodiques. Enfin, elle fournit des indications sur le nombre de pales et le nombre de Mach du rotor.

\section{5 - Références}

[1] M. V. Lowson, J. B. Ollerhead, "A theoretical study of helicopter rotor noise", Journal of Sound and Vibrations, Vol 9, 1969; pp 197-222

[2] P.R. Wallace, "Mathematical analysis of physical problems", 1972

[3] F. Ehrmann, "Contribution à la détection et à la localisation d'hélicoptères par des moyens acoustiques", Thèse de l'Université de Rennes I, Sept. 1993

[4] G. Vezzosi, F. Ehrmann, M. Pécot,"Détection d'hélicoptères par le bruit de leur rotor principal", Colloque GRETSI, Sept. 1993, pp 153-156 